\title{
Oriented Edge Forests for Boundary Detection
}

\author{
Sam Hallman Charless C. Fowlkes \\ Department of Computer Science \\ University of California, Irvine \\ \{shallman, fowlkes\}eics.uci.edu
}

\begin{abstract}
We present a simple, efficient model for learning boundary detection based on a random forest classifier. Our approach combines (1) efficient clustering of training examples based on a simple partitioning of the space of local edge orientations and (2) scale-dependent calibration of individual tree output probabilities prior to multiscale combination. The resulting model outperforms published results on the challenging BSDS500 boundary detection benchmark. Further, on large datasets our model requires substantially less memory for training and speeds up training time by a factor of 10 over the structured forest model. ${ }^{1}$
\end{abstract}

\section{Introduction}

Accurately detecting boundaries between objects and other regions in images has been a long standing goal since the early days of computer vision. Accurate boundary estimation is an important first step for segmentation and detection of objects in a scene and boundaries provide useful information about the shape and identity of those objects. Early work such as the Canny edge detector [6] focused on detecting brightness edges, estimating their orientation [12] and analyzing the theoretical limits of detection in the presence of image noise. However, simple brightness or color gradients are insufficient for handling many natural scenes where local gradients are dominated by fine scale clutter and texture arising from surface roughness and varying albedo.

Modern boundary detectors, such as [19], have emphasized the importance of suppressing such responses by explicit oriented analysis of higher order statistics which are robust to such local variation. These statistics can be captured in a variety of ways, e.g. via textons [16], sparse coding [23], or measures of self-similarity [15]. Such boundary detectors also generally benefit from global normalization provided by graph-spectral analysis [2] or ultra-metric con-

\footnotetext{
${ }^{1}$ This work was supported by NSF DBI-1053036, DBI-1262547, and IIS-1253538
}

sistency [1] which enforce closure, boosting the contrast of contours that completely enclose salient regions.

Recently, focus has turned to methods that learn appropriate feature representations from training data rather than relying on carefully hand-designed texture and brightness contrast measures. For example, [23] learns weightings for each sparse code channel and hypothesized edge orientation while $[8,17]$ predict the probability of a boundary at an image location using a cascade or randomized decision forest built over simple image features. Taking this one step further, the work of [18] and [9] learn not only input features but also the output space using sparse coding or structured-output decision forests respectively. While these approaches haven't yielded huge gains in boundary detection accuracy, they are appealing in that they can adapt to other domains (e.g., learning input features for boundary detection in RGB-D images [23,9] or predicting semantic segmentation outputs [18]). On the other hand, a key difficulty with these highly non-parametric approaches is that it is difficult to control what is going on "under the hood" and to understand why they fail or succeed where they do. Like a fancy new car, they are great when they work, but if ever stranded on a remote roadside, one suddenly discovers there are very few user serviceable parts inside.

In this paper we to take a step back from non-parametric outputs and instead apply the robust machinery of randomized decision forests to the simple task of accurately detecting straight-line boundaries at different candidate orientations and positions within a small image patch. Although this ignores a large number of interesting possibilities such as curved edges and junctions, it should certainly suffice for most small patches of images containing big, smooth objects. We show that such a model, appropriately calibrated and averaged across a small number of scales, along with local sharpening of edge predictions outperforms the best reported results on the BSDS500 boundary detection benchmark.

The rest of the paper is structured as follows. In Section 2, we describe our method for partitioning the space of possible oriented edge patterns within a patch. This leads 

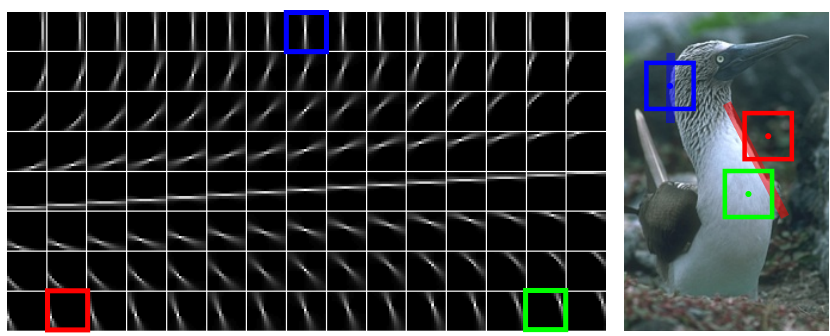

Figure 1: Our boundary detector consists of a decision forest that analyzes local patches and outputs probability distributions over the space of oriented edges passing through the patch. This space is indexed by orientation and signed distance to the edge $(d, \theta)$. These local predictions are calibrated and fused over an image pyramid to yield a final oriented boundary map.

to a simple, discrete labeling over local edge structures. In Section 3, we discuss how to use this discrete labeling to train a random forest to predict edge structure within a patch, and describe a calibration procedure for improving the posterior distributions emitted by the forest. Section 4 then describes how to map the distributions computed over the image into a final, high-quality edge map. Finally, in Section 5 we show experimental results on the BSDS500 boundary detection benchmark.

\section{Clustering Edges}

From a ground truth boundary image, we categorize a $p \times p$ patch either as containing no boundary (background) or as belonging to one of a fixed number of edge categories. A patch is considered background if its center is more than $p / 2$ pixels away from an edge, in which case the patch contains little to no edge pixels.

Non-background patches are distinguished according to the distance $d$ and orientation $\theta$ of the edge pixel closest to the patch center. Thus, patches with $d=0$ have an edge running through the center, and by definition $d$ is never greater than $p / 2$. We choose a canonical orientation for each edge so that $\theta$ lies in the interval $(-\pi / 2, \pi / 2]$. To distinguish between patches on different sides of an edge with the same orientation, we utilized signed distances $d \in(-p / 2, p / 2)$. This yields a parameter pair $(d, \theta)$ for each non-background patch.

Figure 1 shows this two dimensional space of patches. It is worth noting that this space can be given an interesting topology. Since orientation is periodic, a straight edge with parameter $(d, \theta)$ appears identical to one with parameter $(-d, \theta+\pi)$. One can thus identify the top and bottom edges of the space in Figure 1, introducing a half-twist to yield a Möbius strip whose boundary is $\{(d, \theta):|d|=p / 2\} .^{2}$

\footnotetext{
${ }^{2}$ One could also parameterize lines by angle $\theta \in(-\pi, \pi]$ and unsigned
}

From a ground-truth edge map, computing the distance between a patch center and the nearest edge pixel $q$ is straightforward. To be useful, the estimate of $\theta$ should reflect the dominant edge direction around $q$, and be robust to small directional changes at $q$. To accomplish this, we first link all edge pixels in a ground-truth boundary map into edge lists, breaking lists into sublists where junctions occur. We then measure the angle at $q$ by fitting a polynomial to the points around $q$ that are in the same list. In our experiments we use a fitting window of \pm 6 pixels.

Because annotators sometimes attempt to trace out extremely fine detail around an object, boundary annotations will occasionally include very short, isolated "spur" edges protruding from longer contours. Where these occur, estimates of $\theta$ can suffer. We remove all such edges provided that they are shorter than 7 pixels in length. Using standard morphological operations we also fill holes if they exist and thin the result to ensure that all lines are a single pixel thick.

Collecting training data We binned the space of distances $d$ and angles $\theta$ into $n$ and $m$ bins, respectively. Thus every non-background patch was assigned to a discrete label $k$ out of $K=n m$ possible labels. This discrete label space allows for easy application of a variety of off-the-shelf supervised learning algorithms.

In our experiments we used a patch size of $16 \times 16$ pixels, so that distances satisfy $|d|<p / 2=8$. It is natural to set the distance bins one pixel apart, so that $d$ falls into one of $n=15$ bins. Assigning angles $\theta$ to one of $m=8$ bins, leaves $K=120$ edge classes plus background. We chose the orientation binning so that bins 1 and 5 are centered at 90 and 0 degrees respectively, as these orientations are especially common in natural images [22]. Figure 1(a) shows the average ground-truth edge map for all image patches assigned to each of these clusters.

In our experiments we sampled patches uniformly over image locations and over labelings derived from multiple ground-truth segmentations of that image. Since our approach ultimately predicts a $(d, \theta)$ parameter for each non-background image patch, it does not explicitly model patches containing junctions or thin structures involving more than two segments. In practice, such events are relatively rare. In the BSDS500 training dataset, patches containing more than two segments constitute less than $8 \%$ of image patches and only $27 \%$ of all non-background patches. To simplify the learning problem faced by the local classifier, we only utilize patches that contain one or two segments for training.

distance $d \geq 0$. However, this space has a singularity at $d=0$ where patches $(0, \theta)$ and $(0, \theta+\pi)$ are indistinguishable to an edge detector but have different angle parameters. Our parameterization is convenient since it assigns unique coordinates to each line and is smooth everywhere. 


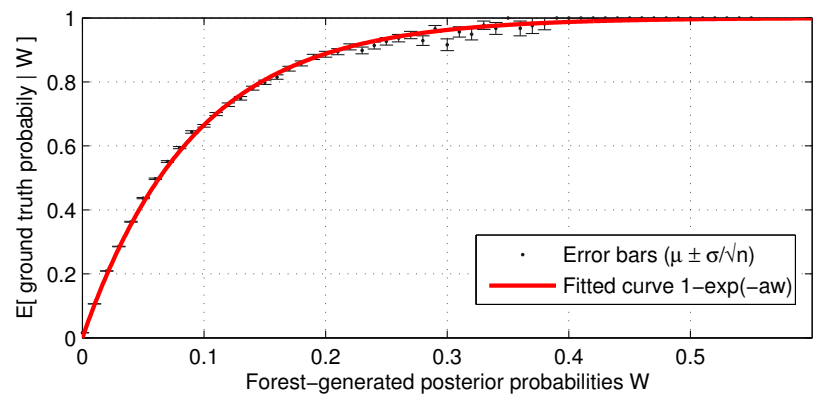

Figure 2: Reliability plot showing the empirical probability of a ground-truth edge label as a function of the score output by the forest computed over a set of validation images. Error bars show standard error in the empirical expectation. Red curve shows a simple functional fit $1-\exp (-\beta w)$ which appears to match the empirical distribution well. We use this estimated function (one scalar parameter $\beta$ per scale) to calibrate the distribution of scores over different edges $(d, \theta)$ predicted by the forest. Performing this calibration prior to combining and compositing predictions across scales improves final performance.

\section{Oriented Edge Forest}

Using the labeling procedure outlined in Section 2, we can build a training dataset comprised of color image patches $\mathbf{x}$, each with a corresponding edge cluster assignment $\mathbf{y} \in\{0,1, \ldots, K\}$ where $K$ is the number of edge clusters and $\mathbf{y}=0$ represents the background or "no boundary" class. Inspired by the recent success of random decision forests for edge detection [17, 9], we train a random forest classifier to learn a mapping from patches to this label set. In this section we discuss forest training and calibration procedures that yield high-quality edge probability estimates.

Randomized Decision Forests Random forests are a popular ensemble method in which randomized decision trees are combined to produce a strong classifier. Trees are made random through bagging and/or randomized node optimization [7], in which the binary splits at the nodes of the tree are limited to using only a random subset of features.

In our framework, the output label space predicted by the forest is a small discrete set ( $K$ possible edge orientations and locations relative to the center of the patch or background) and may be treated simply as a k-way classification problem. When training a given decision tree, features are selected and split thresholds are chosen to optimize the Gini impurity measure [5]. In practice we find that the particular choice of class purity metric does not have a noticeable impact on performance. We did find it important to have bal- anced training data across classes and used an equal number training examples per class.

Image Features We adopt the same feature extraction process used in [9]. In this approach, images are transformed into a set of feature channels, and the descriptor for a patch is computed simply by cropping from the corresponding window in the array of feature channels. These features are comprised of color and gradient channels, and are downsampled by a factor of 2. Binary splits performed at the tree nodes are accomplished by thresholding either a pixel read from a channel or the difference between two pixels from the same channel. See [9] for full details.

Ensemble Averaging Equipped with a forest trained to recognize oriented edge patterns, the next step is to apply the forest over the input image. We have found that the details of how we fuse the predictions of different trees can have a significant effect on performance. Two standard approaches to combining the output of a ensemble of classifiers are averaging and voting.

For a given test image patch $\mathbf{x}$, each individual tree $t$ produces an estimate $p_{t}(k \mid \mathbf{x})$ of the posterior distribution over the $K+1$ class labels based on the empirical distribution observed during training. We would like to combine these individual estimates into a final predicted score vector $\mathbf{w}(k \mid \mathbf{x})$. The most obvious way to combine the tree outputs is averaging

$$
\mathbf{w}(k \mid \mathbf{x})=\frac{1}{T} \sum_{t=1}^{T} p_{t}(k \mid \mathbf{x}), \quad k=1, \ldots, K
$$

An alternative, often used for ensembles of classifiers which only output class labels instead of posteriors is voting

$$
\mathbf{w}(k \mid \mathbf{x})=\frac{1}{T} \sum_{t=1}^{T} \mathbf{1}_{\left[k=\arg \max _{k} p_{t}(k \mid \mathbf{x})\right]}
$$

where $\mathbf{1}$ is the indicator function.

In general, we find that averaging provides somewhat better detection accuracy than voting, presumably because the votes carry less information than the full posterior distribution (see Section 5). One disadvantage of averaging is that it requires one to maintain in memory all of the empirical distributions $p$ at every leaf of every tree. Voting not only requires less storage for the forest but also reduces runtime. Constructing $\mathbf{w}$ via averaging requires $O(K T)$ while voting only requires $O(T)$. The resulting $\mathbf{w}$ is also sparse which can lead to substantial speed improvements in the edge fusion steps described below (Section 4). Voting may thus be an efficient alternative for time-critical applications. 
Calibration In order to fuse edge predictions across different scales within an image and provide boundary maps whose values can be meaningfully compared between images, we would like the scores $\mathrm{w}$ to be accurately calibrated. Ideally the scores $\mathbf{w}$ output for a given patch would be the true posterior probability over edge types for that patch. Let $\mathbf{x}$ be a patch sampled from the dataset and $y$ the true edge label for that patch. If the scores $\mathbf{w}(k \mid \mathbf{x})$ output by the classifier are calibrated then we would expect that

$$
P(y=k \mid \mathbf{w}(k \mid \mathbf{x})=s)=s
$$

To evaluate calibration, we extracted a ground-truth label indicator vector for every labeled image patch in a held-out set of validation patches $\left\{\left(\mathbf{x}_{i}, y_{i}\right)\right\} .{ }^{3}$ We then computed the empirical expectation of how often a particular label $k$ was correct for those patches that received a particular score $s$.

$$
P(y=k \mid \mathbf{w}(k \mid \mathbf{x})=s) \approx \frac{1}{|B(k, s)|} \sum_{i \in B(k, s)} \mathbf{1}_{\left[y_{i}=k\right]}
$$

where

$$
B(k, s)=\{i: \mathbf{w}(k \mid \mathbf{x}) \in[s \pm \epsilon]\}
$$

is a bin of width $2 \epsilon$ centered at $s$.

Figure 2 shows the resulting reliability plot, aggregated over non-background patches. Results were very similar for individual edge labels. While one might expect that a forest trained to minimize entropy of the posterior predictions would tend to be overconfident, we found that the forest average scores for non-background patches actually tended to underestimate the true posterior! This remained true regardless of whether we used voting or averaging.

Previous work has used logistic regression in order to calibrate classifier output scores [20]. For the oriented edge forest, we found that this miscalibration for nonbackground labels is much better fit by an exponential

$$
\hat{\mathbf{w}}(k \mid \mathbf{x})=f_{\beta}(\mathbf{w}(k \mid \mathbf{x}))=1-\exp (-\beta \mathbf{w}(k \mid \mathbf{x}))
$$

where $\beta$ is a scalar. We fitted this function directly to the binary indicator vectors $\mathbf{1}_{\left[y_{i}=k\right]}$ rather than binned averages in order to give equal weight to each training example.

We also explored a wide variety of other calibration models including sigmoid-shaped functions such as tanh, richer models that fit an independent parameter $\beta_{k}$ per class label, and joint calibration across all class labels. We even considered a non-parametric approach in which we treated the 120-D ground truth label vectors as structured labels and trained an additional structured random forest [11]. We found that using a single scalar $\beta$ for all non-background

\footnotetext{
${ }^{3}$ When multiple humans segmentations generated conflicting labels for the patch, we averaged them to produce a "soft" non-binary label vector.
}

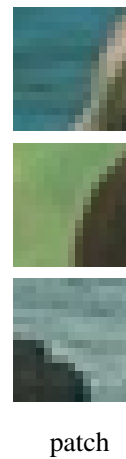

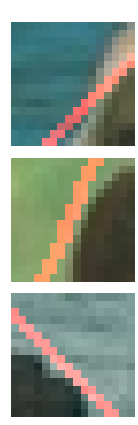

$\mathrm{sh}=0$
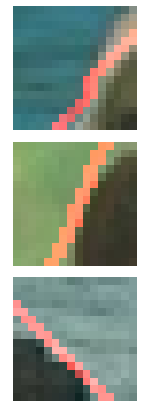

$\mathrm{sh}=1$
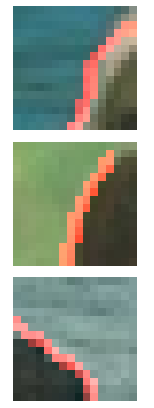

$\mathrm{sh}=2$
Figure 3: Examples of sharpening a single predicted edge patch label based on underlying image evidence. The patch is resegmented based on the initial straight edge label (2nd column) by reassigning pixels near the boundary to the region with more similar mean RGB value.

scores is highly efficient ${ }^{4}$ and performed as well as any calibration scheme we tried. When performing multiscale fusion (Section 4), we fit a distinct $\beta$ for each scale, the values of which typically ranged from 6 to 10 .

\section{Edge Fusion}

Having applied the forest over the input image, we are left with a collection of calibrated probability estimates $\hat{\mathbf{w}}$ at every spatial position. Because these distributions express the likelihood of both centered $(d=0)$ as well as distant, off-center $(d \neq 0)$ edges, the probability of boundary at a given location is necessarily determined by the tree predictions over an entire neighborhood around that location. In this section, we describe how to resolve these probabilities into a single, coherent image of boundary strengths. The end result will be an oriented signal $E(x, y, \theta)$ that specifies the probability of boundary at location $(x, y)$ in the binned direction $\theta$.

Edge sharpening By focusing on oriented lines, our detector is trained to recognize coarse edge statistics but cannot predict more detailed structure, e.g. local curvature or wiggles of a few pixels in a contour. As the size of the analyzed patch increases relative to the size of an object, the straight line assumption becomes a less accurate representation of the shape. In order to provide a more detailed prediction of the contour shape, we utilize a local segmentation procedure similar to the sharpening method introduced by Dollár and Zitnick [11]. This is similar in spirit to the notion of "Edge Focusing" [4] in which coarse-to-fine tracking

\footnotetext{
${ }^{4}$ For a sparse voting implementation, one can do nearly as well using the fast approximation $f(\mathbf{w})=\min \{1, \mathbf{w}\}$.
} 
utilizes edge contrast measured at a coarse scale but contour shape derived from fine scale measurements.

Consider a hypothesized (straight) edge predicted by the forest at a given location. We compute the mean RGB color of the pixels on each side of the hypothesized edge inside a $16 \times 16$ pixel patch centered at the location. We then resegment pixels inside the patch by assigning them to one of these two cluster means. To prevent the local segmentation from differing wildly with the original oriented line predicted by the forest, we only reassign pixels within 1 or 2 pixels distance from the hypothesized segment boundary. We will use the notation $M_{(x, y, k)}(i, j)$ to denote the sharpened binary edge mask of type $k=(d, \theta)$ computed for a patch centered at location $(x, y)$ in an input image. Figure 3 shows examples of individual patches along with the resulting mask $M$ for more and less aggressive sharpening.

Compositing Given local estimates of the likelihood (calibrated scores $\hat{\mathbf{w}}$ ) and precise boundary shapes (sharpened masks $M$ ) for each image patch, we predict whether a location $(x, y)$ is on a boundary by averaging over patch predictions for all patches that include the given location. Using the convention that $M_{(x, y, k)}(0,0)$ is the center of a given edge mask and indexing $\hat{\mathbf{w}}$ by the coordinates of each patch in the image, we can write this formally as

$E(x, y, \theta)=\sum_{k \in\{(d, \theta) \forall d\}} \sum_{(i, j) \in O_{x y}} \hat{\mathbf{w}}(i, j, k) M_{(i, j, k)}(x-i, y-j)$

where $O_{x y}$ are the coordinates of patches overlapping $x, y$ and $k$ ranges over all predicted labels which are compatible with orientation $\theta .^{5}$

Combining multiple scales The compositing procedure in the previous section can easily be repeated to produce an $E(x, y, \theta, s)$ for different scaled versions of an input image. In general, combining results at different scales is known to improve performance [21]. We apply the detector at four scales. To detect large-scale edge structure we run at scales $s=1 / 4,1 / 2$. We find that at these resolutions heavy sharpening is less desirable (see Figure 4). Finer edge structure is discovered at scales $s=1,2$, and at these scales more aggressive sharpening is preferred. The results are averaged to produce a final output, as in [9]. The strengths of each scale can be seen in benchmark results in Figure 8, where the curves tend toward higher precision and lower recall as $s$ decreases. It is interesting to note that including $s=2$ is beneficial despite being dominated everywhere by $s=1$. As lower scales are added, precision increases but asymptotic recall suffers. Including scale 2 allows us to maintain the benefits of low scales without the loss in recall.

\footnotetext{
${ }^{5}$ Note that if we do not perform any sharpening on the edge masks, then $M$ is the same at every image location $(i, j)$ and the resulting operation is simply a correlation of $\hat{\mathbf{w}}$ with $M$ summed over channels $k$.
}

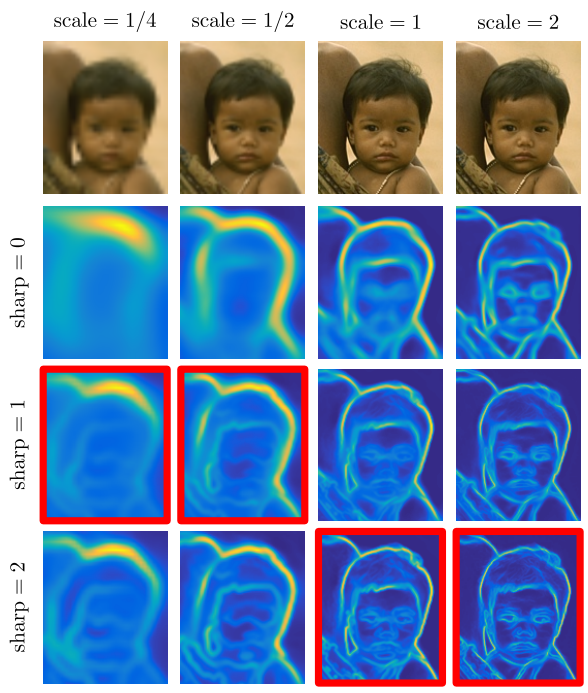

Figure 4: The output of the forest when run at different scales (by down/up-sampling the input image and with different degrees of edge sharpening). Running the forest on a low-resolution version of the image yields blurry detections that respond to coarse scale image structure. Sharpening allows the spatial localization of this broad signal and alignment of predictions made by overlapping local classifiers. We found that coarse scale information is best utilized by performing only modest sharpening of the lowestresolution output to allow strong, broad edge responses to combine with finely localized edges from higher scales.

\section{Experiments}

\subsection{Benchmark Performance}

Figure 5 shows the performance of our model on the BSDS500 test set over the full range of operating thresholds. Our system outperforms existing methods in the high precision regime, and is virtually identical to SE [11] at high recall. Table 1 lists quantitative benchmark results and compares them to recently published methods.

Regions We combine OEF with MCG [3] to produce segmentation hierarchies from our edge detector output. MCG originally used contour strengths from SE, and we found its implementation is sensitive to the statistics of SE output. Rather than tune the implementation, we simply applied a monotonic transformation of our detector output to match the SE distribution (see Section 5.2). The resulting combination, denoted OEF+MCG in Figure 5 and Table 1, is surprisingly effective, attaining an ODS of 0.76 on BSDS.

Diagnostic experiments The performance benefits of calibration are shown in Table 2. Calibration results in a clear improvement below $50 \%$ recall, boosting average precision from 0.81 to 0.82 . In the same table we also report 


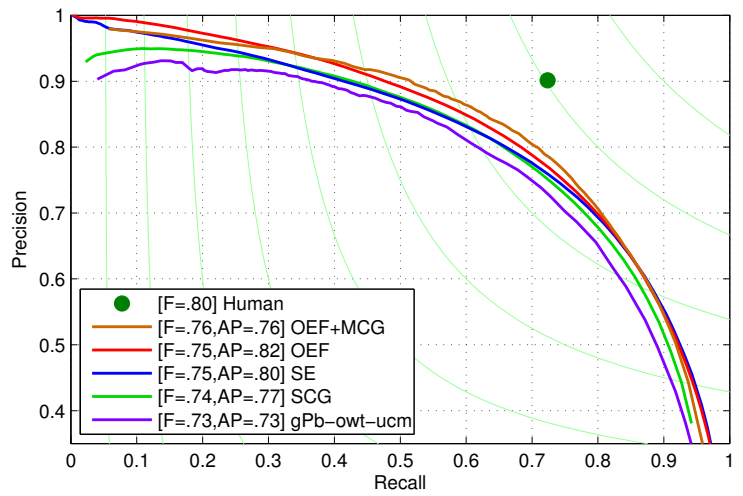

Figure 5: Results on BSDS500. Our system outperforms existing methods in the high precision regime, and is virtually identical to SE at high recall.

benchmark scores for our model when predictions from the ensemble are combined by voting (Eqn 2) rather than averaging. Voting appears to match averaging up to roughly $20 \%$ recall, beyond which it falls behind.

Amount of training data We find that our model benefits significantly from large amounts of training data. In Figure 7, we show how performance on BSDS500 varies as the amount of patches used for training is increased. Important for utilizing large datasets is efficient training. We discuss timing details in Section 5.3.

\subsection{Visualizing Detector Output}

Qualitative results on a selection of test images are shown in Figure 9. Notice that although the forest is trained only to detect straight edges, its performance at corners and junctions is as good as any other method.

One difficulty with visualizing boundary detector outputs is that monotonic transformations of the output boundary maps do not affect benchmark performance but can dramatically affect the qualitative perception of boundary quality. A consequence of this is that qualitative comparisons of different algorithms can be misleading, as the most salient differences tend not to be relevant to actual performance. An example of this can be seen in Figure 6(c), which shows the raw output of SCG [23] on a test image. Comparing this against the results of other algorithms, the output appears overly dim and gives the impression that SCG is relatively insensitive to all but the most obvious edges. However, a quick glimpse at its precision-recall curve reveals that this impression is false.

To visualize boundary detector outputs in a way that highlights relevant differences but removes these nuisance factors without affecting benchmark results, we determine a global monotonic transformation for each boundary detector which attempts to make the average histogram of re-

\begin{tabular}{l|ccc} 
& ODS & OIS & AP $^{6}$ \\
\hline Human & .80 & .80 & \\
\hline gPb [2] & .71 & .74 & .65 \\
gPb-owt-ucm [2] & .73 & .76 & .73 \\
Sketch Tokens [17] & .73 & .75 & .78 \\
SCG [23] & .74 & .76 & .77 \\
DeepNet [14] & .74 & .76 & .76 \\
PMI [13] & .74 & .77 & .78 \\
SE [11] & .75 & .77 & .80 \\
SE + MCG [3] & .75 & .78 & .76 \\
\hline OEF & .75 & .77 & $\mathbf{. 8 2}$ \\
OEF + MCG & $\mathbf{. 7 6}$ & $\mathbf{. 7 9}$ & .76 \\
\hline & & &
\end{tabular}

Table 1: Benchmark scores on BSDS500.

sponse values across all images match a standard distribution. We first choose a reference algorithm (we used SE) and compute its histogram of responses over an image set to arrive at a target distribution. For every boundary map produced by another algorithm we compute a monotonic transformation for that boundary map that approximately matches its histogram to the target distribution. Averaging these mappings produces a single monotonic transformation specific to that algorithm which we use when displaying outputs.

\subsection{Computational Costs}

A key advantage of our simplified approach relative to SE [11] is significantly reduced resources required at training time. We report training times for both systems assuming each tree is trained on its own bootstrap sample of $4 \times 10^{6}$ patches.

Training For both models, the data sampling stage takes $\sim 20$ minutes per tree. Because we expose the trees to smaller random feature sets, this takes approximately 15 gigabytes (GB) of memory, compared to $33 \mathrm{~GB}$ for SE. To train on this much data, SE takes over 3.25 hours per tree and requires about $54 \mathrm{~GB}$ of memory. This is due to the per-node discretization step, where at every tree node PCA is applied to descriptors derived from the training examples at that node. In contrast, our approach is almost $40 \times$ faster, taking about 5 minutes per tree, with memory usage at roughly $19 \mathrm{~GB}$.

Detection We report runtimes for images of size $480 \times$ 320 on an 8-core Intel i7-950. A voting implementation of our system (Eqn 2) runs in about 0.7 seconds per image, compared to 0.4 seconds for SE. Runtime increases to 2 seconds when using averaging (Eqn 1).

\footnotetext{
${ }^{6}$ We note that the lower AP for MCG is because the benchmark computes average precision over the interval $[0,1]$ but the precision-recall curve does not extend to 0 recall. Monotonically extending the curve to the left (e.g., as is done in PASCAL VOC) yields AP values of gPb-owt$\mathrm{ucm}=0.76, \mathrm{SCG}=0.78, \mathrm{SE}+\mathrm{MCG}=0.81, \mathrm{OEF}+\mathrm{MCG}=0.82$
} 


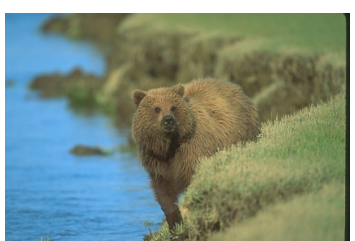

(a) Original image

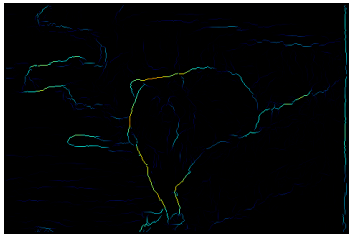

(c) SCG original

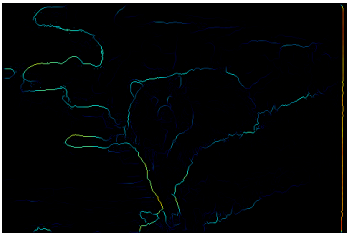

(e) SE original

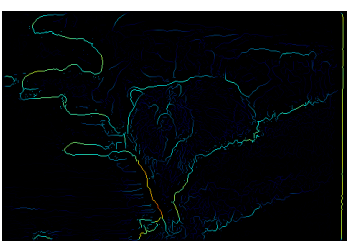

(g) OEF original

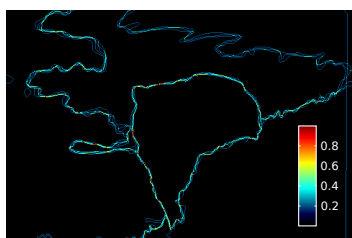

(b) Ground truth

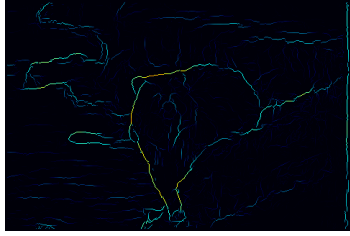

(d) SCG new

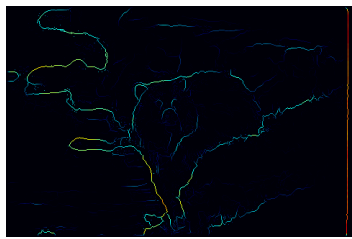

(f) SE new

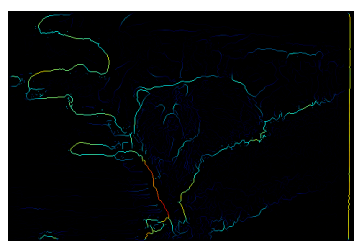

(h) OEF new
Figure 6: We compute a monotonic transformation for each algorithm which attempts to make the distributions of output values as similar as possible. This serves to highlight relevant differences by making the visualizations of different algorithms invariant to monotonic transformations that don't effect benchmark performance. Here we show the effect of normalizing all three algorithms to match the average SE distribution. Note that this even changes the appearance of SE since its distribution on a particular image will differ from its average distribution over all images.

The primary reason that averaging is slower is it requires more time for edge sharpening since the predicted score vectors $\mathbf{w}$ are not sparse. To reduce the amount of computation spent on sharpening, we leverage the following observation. The same oriented edge will appear at different offsets $d$ across neighboring windows. The weights $\mathbf{w}$ for a given orientation can thus all be aligned (e.g., with the $d=0$ channel) by simple translation and summed prior to sharpening. Thus the collection of 120-dimensional distributions computed over the image are "collapsed" down to 8 dimensions, one per orientation. This optimization reduces runtime from 11 seconds down to just 2 seconds, while dropping ODS and AP by less than 0.003 .

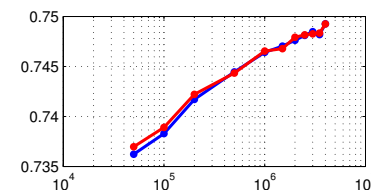

(a) ODS

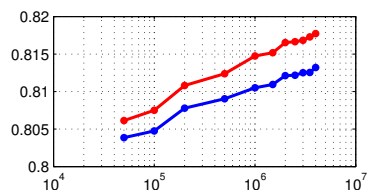

(b) AP
Figure 7: Performance on BSDS500 as a function of the number of training examples, before calibration (blue) and after calibration (red). The smallest model was trained on $5 \times 10^{4}$ examples and the largest on $4 \times 10^{6}$ examples. Training times vary from less than one minute (40 seconds data collection +6 seconds tree training) per tree for the smallest model to under 30 minutes (15-20 minutes data collection +5 minutes tree training) per tree for the largest model.

\begin{tabular}{l|ccc} 
& ODS & OIS & AP \\
\hline vote & .74 & .77 & .80 \\
average & .75 & .77 & .81 \\
\hline vote+cal & .75 & .77 & .81 \\
average+cal & .75 & .77 & .82 \\
+ sharp $=2,2,2,2$ & .75 & .77 & .81 \\
+ sharp $=1,1,1,1$ & .75 & .77 & .81 \\
+ sharp $=0,0,0,0$ & .74 & .77 & .78 \\
\hline
\end{tabular}

Table 2: We analyze different variants of our system on BSDS. We use the notation "sharp=a,b,c,d" to indicate the sharpening levels used for scales $1 / 4,1 / 2,1,2$, respectively. All algorithms use sharpen $=1,1,2,2$ unless otherwise stated. Rows 1-2 compare voting (Eqn 2) and averaging (Eqn 1) prior to calibration, showing that having trees emit full distributions over labels is more powerful than casting single votes. Rows 3-4 show that calibration improves performance. The last four rows correspond to the calibrated model with different sharpening levels, and show that it helps to do less sharpening at lower scales.

\section{Discussion}

In many ways our oriented edge forest is similar to SCG in that we train a classifier which predicts the boundary contrast at each hypothesized edge orientation. A chief difference is the addition of the $d$ parameter which allows the classifier to make useful predictions even when it is not centered directly over an edge. For a traditional detector, points near a boundary also tend to have high contrast but it is unclear whether they should constitute positive or negative examples, and such training data is often discarded.

Our proposed system is also quite similar to SE and Sketch Tokens (it uses the same features, choice of classifier, etc.). We find it interesting that the inclusion of other 


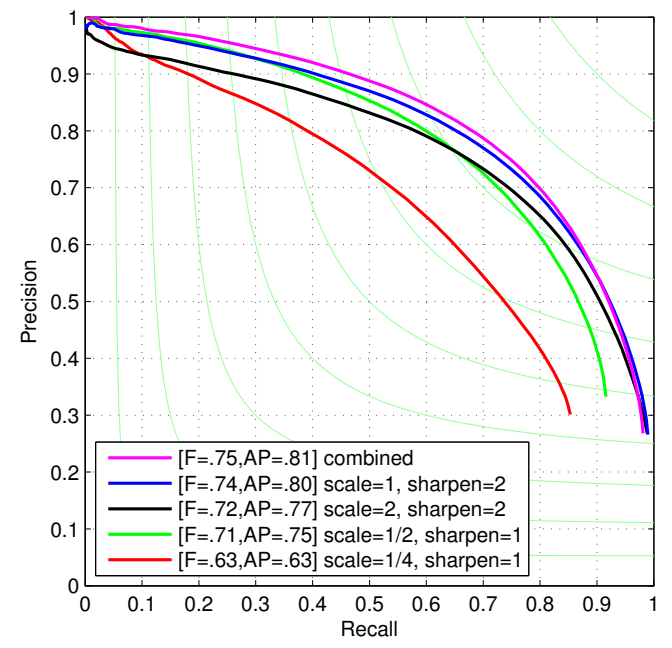

Figure 8: Results on BSDS showing the performance of our algorithm when run at a particular scale, compared to the results after multiscale combination. No calibration is performed here. Consistent with the findings of [21], the combined model greatly outperforms any fixed-scale model.

types of output, such as junctions or parallel edges, is not necessary. Such events are quite rare, so there is probably not enough training data to really learn the appearance of more complicated local segmentations. In fact we found that training SE without complex patches ( $>2$ segments) worked just as well.

A final observation is that having the classifier output patches may not be necessary. It is certainly computationally advantageous since a given pixel receives votes from many more trees, but given enough trees, we find that Sketch Tokens performs essentially as well when only predicting the probability at the center pixel. This suggests that the real value of structured outputs for edge detection is in partitioning the training data in a way that simplifies the task of the decision tree: breaking patches into different clusters allows the tree to learn the appearance of each cluster separately rather than having to discover the structure by mining through large quantities of data. We hypothesize that other types of supervisory information-e.g. curvature, depth of a surface from the camera, change in depth across an edge, figure-ground orientation of a contour, material or object category of a surface-may further simplify the job of the forest, allowing it to better fit the data more readily than simply training on a larger set of undistinguished patches.

\section{References}

[1] P. Arbelaez. Boundary extraction in natural images using ultrametric contour maps. In Computer Vision and Pattern Recognition Workshop, 2006. CVPRW'06. Conference on, pages 182-182. IEEE, 2006.
[2] P. Arbelaez, M. Maire, C. Fowlkes, and J. Malik. Contour detection and hierarchical image segmentation. IEEE PAMI, 33(5), 2011. 1, 6, 10

[3] P. Arbeláez, J. Pont-Tuset, J. T. Barron, F. Marques, and J. Malik. Multiscale combinatorial grouping. CVPR, 2014. 5, 6, 10

[4] F. Bergholm. Edge focusing. Pattern Analysis and Machine Intelligence, IEEE Transactions on, (6):726-741, 1987. 4

[5] L. Breiman, J. Friedman, C. J. Stone, and R. A. Olshen. Classification and regression trees. CRC press, 1984. 3

[6] J. Canny. A computational approach to edge detection. Pattern Analysis and Machine Intelligence, IEEE Transactions on, (6):679-698, 1986. 1

[7] A. Criminisi and J. Shotton. Decision forests for computer vision and medical image analysis. Springer, 2013. 3

[8] P. Dollar, Z. Tu, and S. Belongie. Supervised learning of edges and object boundaries. In Computer Vision and Pattern Recognition, 2006 IEEE Computer Society Conference on, volume 2, pages 1964-1971. IEEE, 2006. 1

[9] P. Dollár and C. L. Zitnick. Structured forests for fast edge detection. In Computer Vision (ICCV), 2013 IEEE International Conference on, pages 1841-1848. IEEE, 2013. 1, 3, 5

[10] P. Dollár and C. L. Zitnick. Fast edge detection using structured forests. arXiv preprint arXiv:1406.5549, 2014. 10

[11] P. Dollár and C. L. Zitnick. Fast edge detection using structured forests. PAMI, 2015. 4, 5, 6, 9

[12] W. T. Freeman and E. H. Adelson. The design and use of steerable filters. IEEE Transactions on Pattern analysis and machine intelligence, 13(9):891-906, 1991. 1

[13] P. Isola, D. Zoran, D. Krishnan, and E. H. Adelson. Crisp boundary detection using pointwise mutual information. In Computer VisionECCV 2014, pages 799-814. Springer, 2014. 6

[14] J. J. Kivinen, C. K. Williams, N. Heess, and D. Technologies. Visual boundary prediction: A deep neural prediction network and quality dissection. In Proceedings of the Seventeenth International Conference on Artificial Intelligence and Statistics, pages 512-521, 2014. 6

[15] M. Leordeanu, R. Sukthankar, and C. Sminchisescu. Generalized boundaries from multiple image interpretations. 2012. 1

[16] T. Leung and J. Malik. Representing and recognizing the visual appearance of materials using three-dimensional textons. International Journal of Computer Vision, 43(1):29-44, 2001. 1

[17] J. J. Lim, C. L. Zitnick, and P. Dollár. Sketch tokens: A learned midlevel representation for contour and object detection. In Computer Vision and Pattern Recognition (CVPR), 2013 IEEE Conference on, pages 3158-3165. IEEE, 2013. 1, 3, 6

[18] M. Maire, S. X. Yu, and P. Perona. Reconstructive sparse code transfer for contour detection and semantic labeling. In Asian Conference on Computer Vision (ACCV), 2014. 1

[19] D. R. Martin, C. C. Fowlkes, and J. Malik. Learning to detect natural image boundaries using local brightness, color, and texture cues. Pattern Analysis and Machine Intelligence, IEEE Transactions on, 26(5):530-549, 2004. 1

[20] J. C. Platt. Probabilistic outputs for support vector machines and comparisons to regularized likelihood methods. In Advances in large margin classifiers. Citeseer, 1999. 4

[21] X. Ren. Multi-scale improves boundary detection in natural images. In Computer Vision-ECCV 2008, pages 533-545. Springer, 2008. 5 , 8

[22] A. Torralba and A. Oliva. Statistics of natural image categories. Network: computation in neural systems, 14(3):391-412, 2003. 2

[23] R. Xiaofeng and L. Bo. Discriminatively trained sparse code gradients for contour detection. In Advances in neural information processing systems, pages 584-592, 2012. 1, 6, 9 


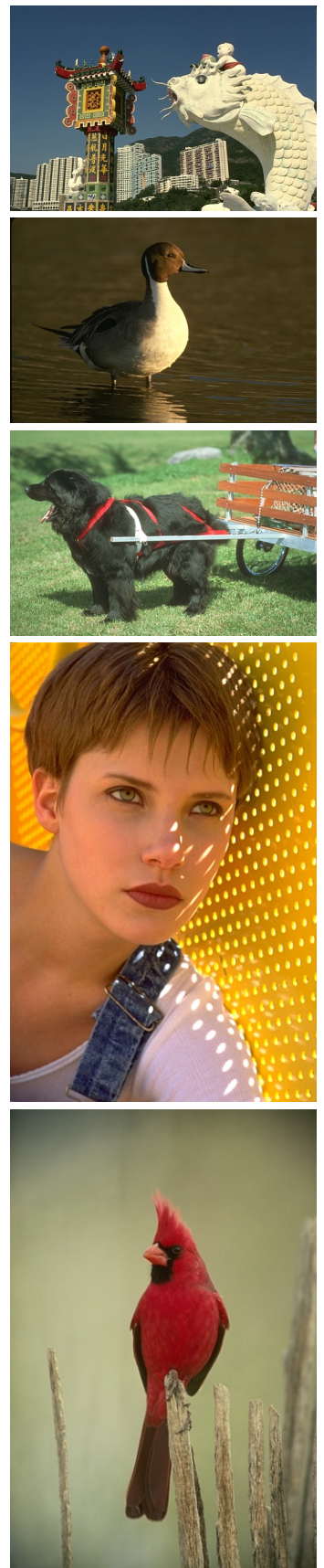

(a) Original image
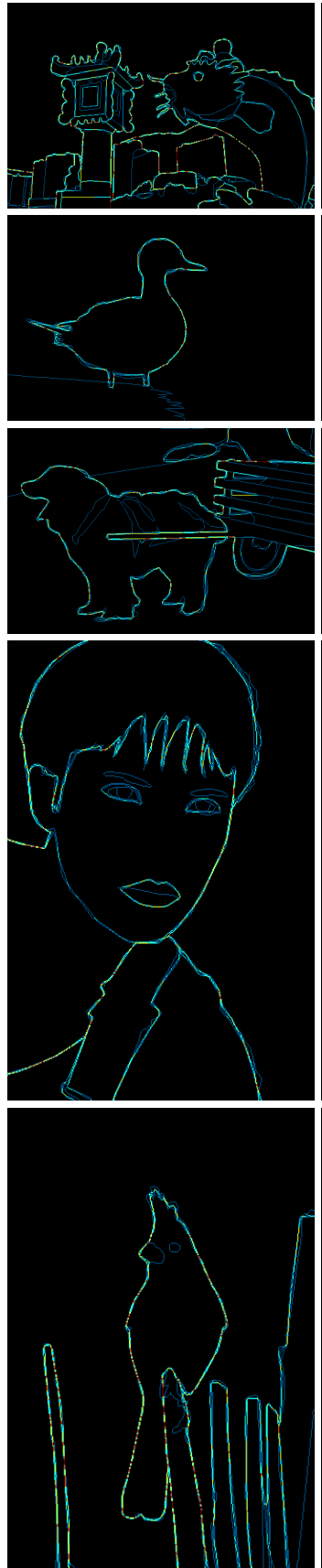

(b) Ground truth
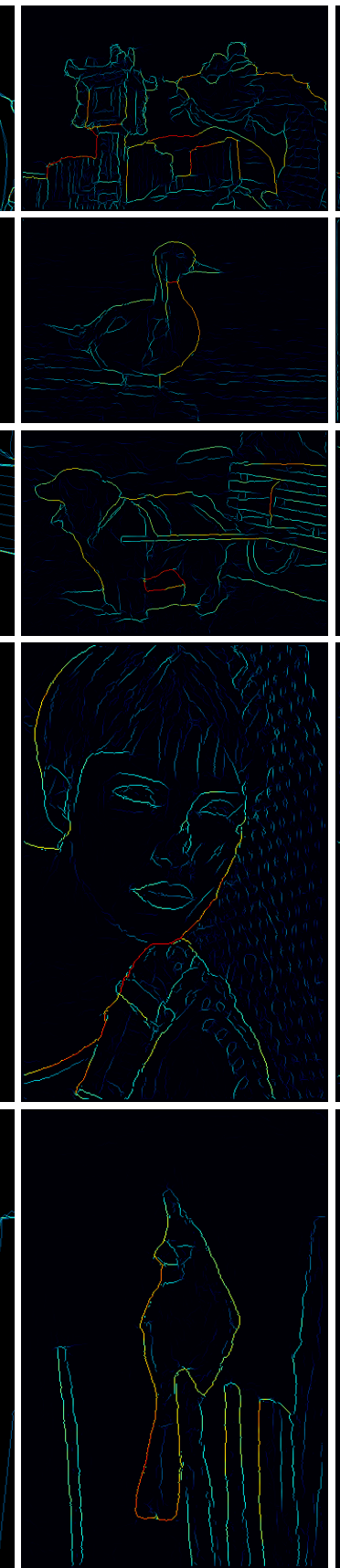

(c) SCG [23]
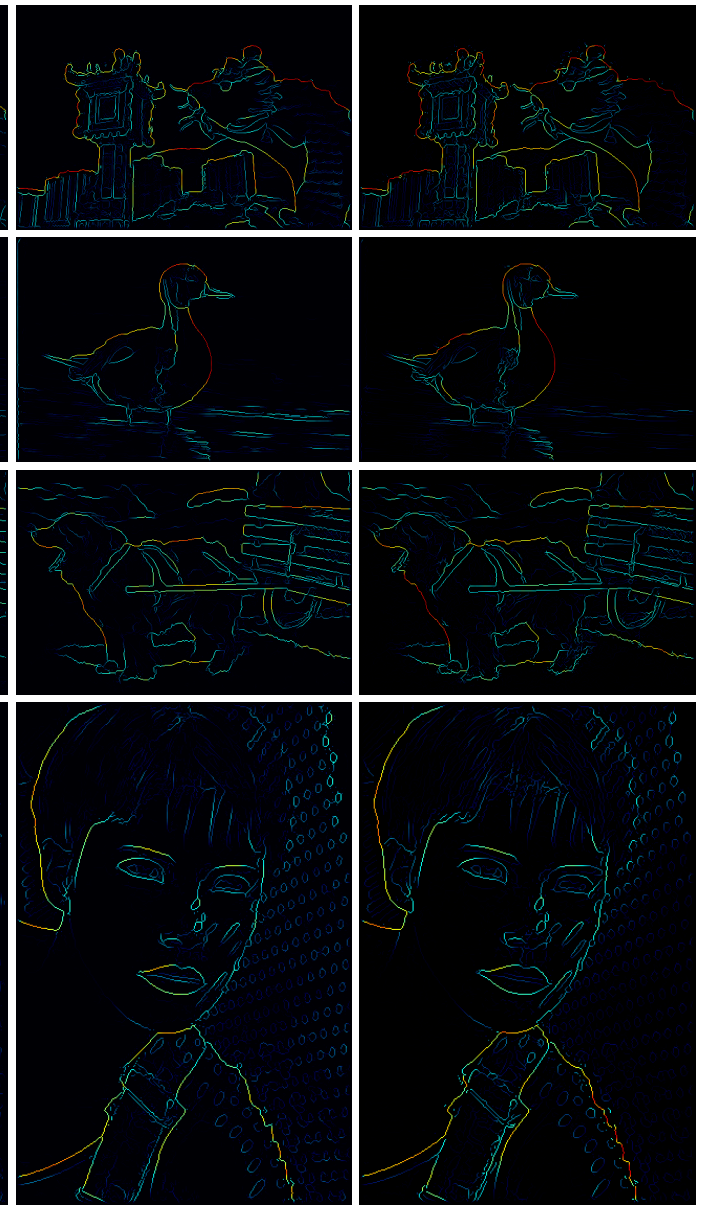

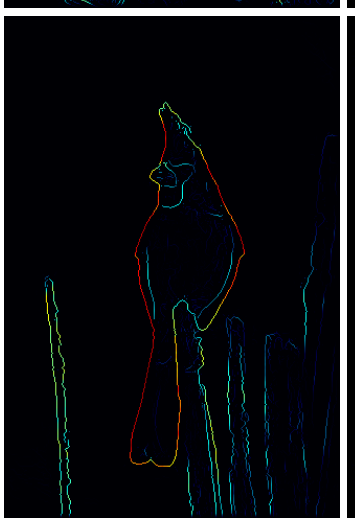

(d) SE [11]

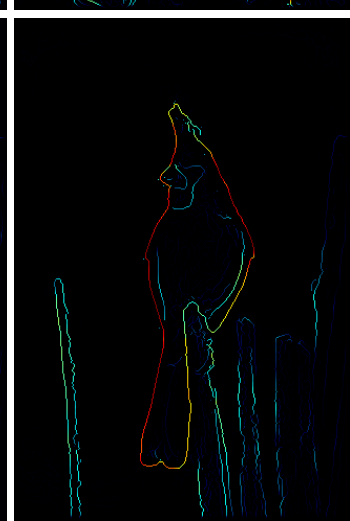

(e) $\mathrm{OEF}$

Figure 9: Example results on the BSDS test set after non-maximal suppression. Rows 1,4 demonstrate our model correctly suppressing edges belonging to background texture, such as on the scales on the statue and the dots around the woman's face. Also note that in row 2 our results show significantly less weight on the false edges along the surface of the water. To allow for meaningful visual comparisons, we derive a global monotonic transformation for each algorithm that attempts to make the distributions of output values the same across all algorithms. This post-processing step preserves the relative ordering of the edges, so benchmark results are unaffected but some irrelevant differences are eliminated from the boundary map visualization. Details can be found in Section 5.2. 


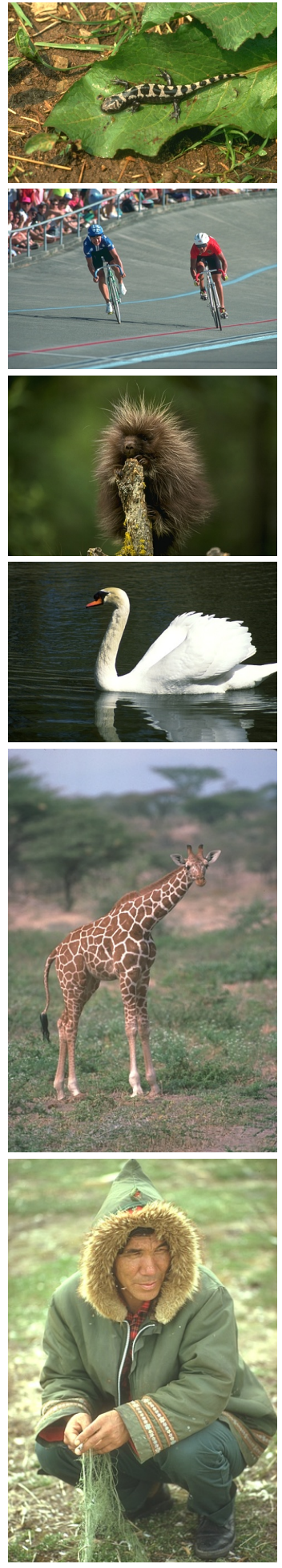

(a) Original image

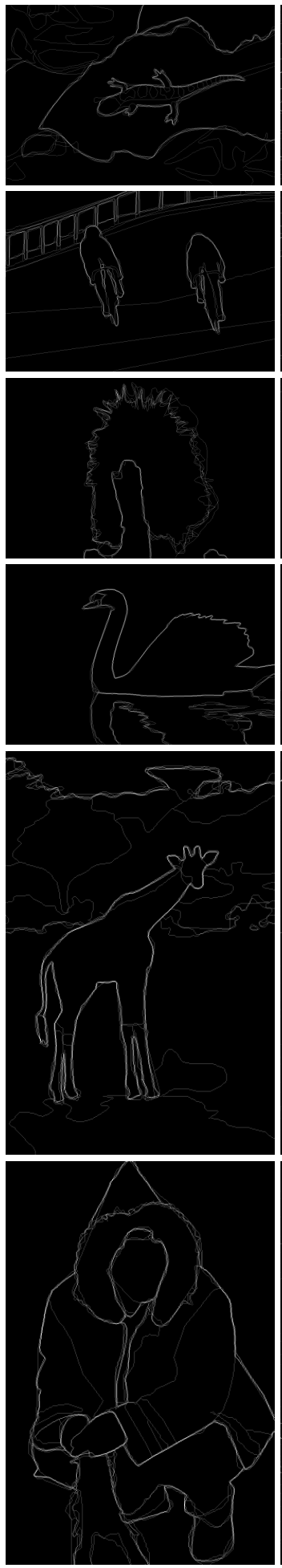

(b) Ground truth
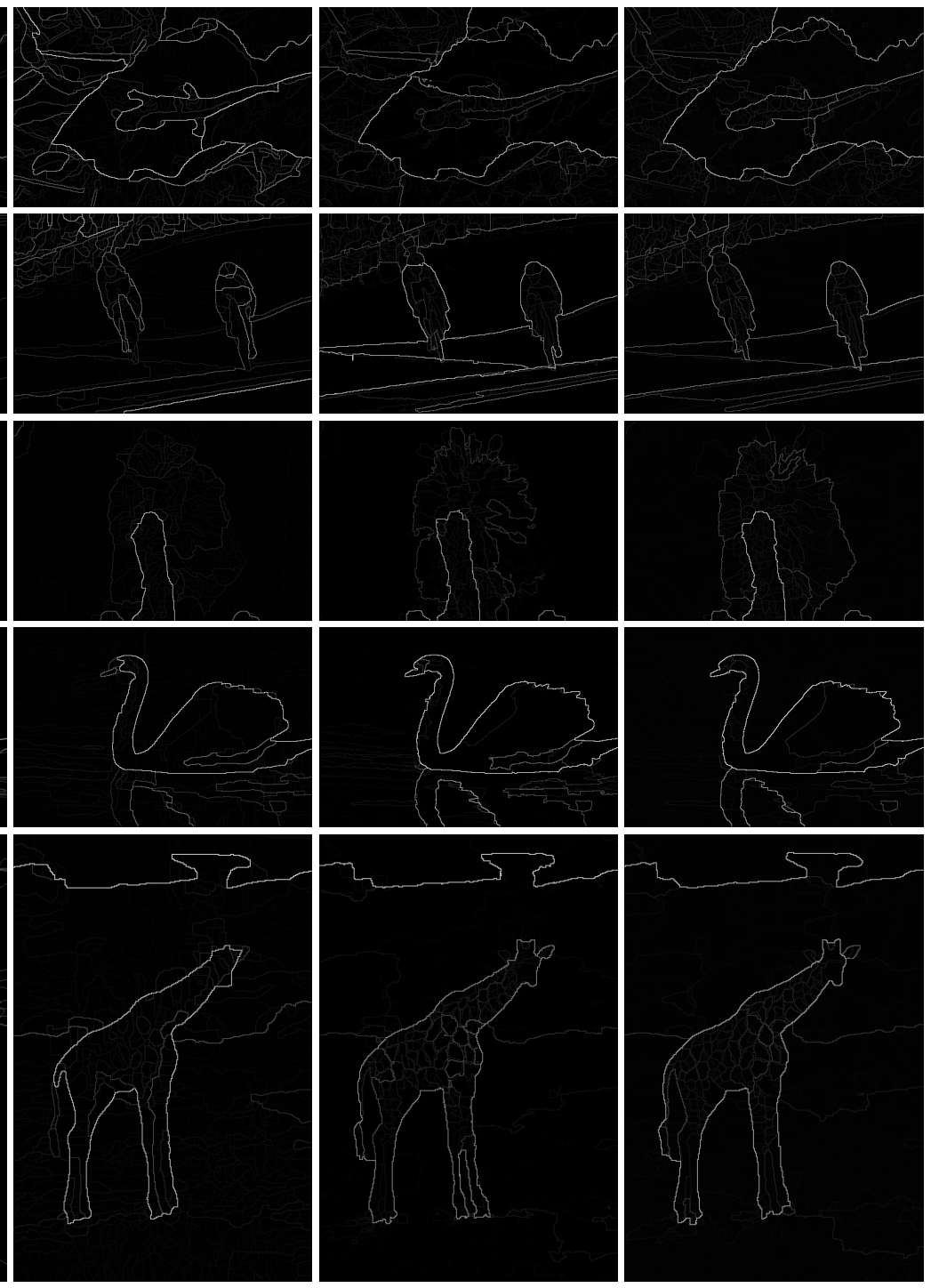

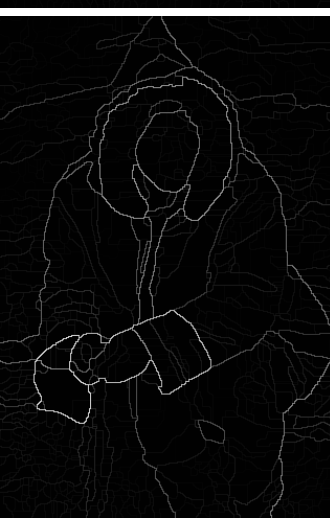

(c) gPb-owt-ucm [2]

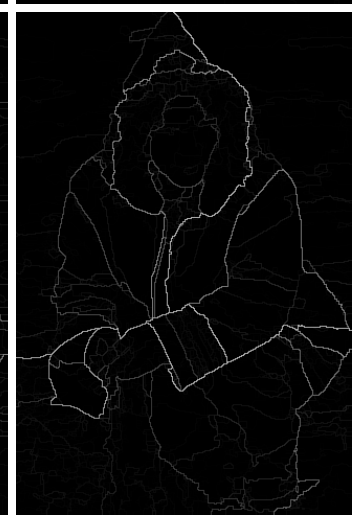

(d) SE [10] + MCG [3]

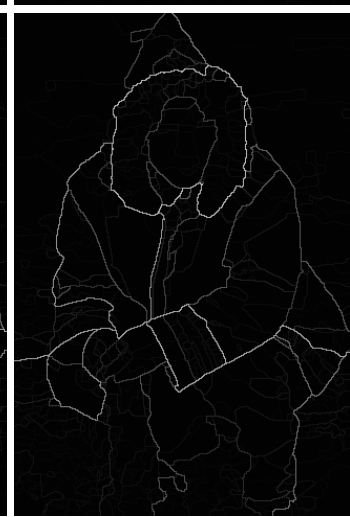

(e) OEF + MCG [3]

Figure 10: Segmentation hierarchies for a selection of BSDS test images, represented here as ultrametric contour maps [2]. We found the combination of OEF and MCG [3] to produce high-quality segmentations that benchmark well on BSDS, achieving an F-measure of 0.76. Details of this pipeline are given in Section 5.1 (Regions). As in Figure 9, results are visualized according to the procedure described in Section 5.2. 